\author{
MITSUBISHI ELECTRIC RESEARCH LABORATORIES \\ http://www.merl.com
}

\title{
An Improved Representation for Stroke-based Fonts
}

\author{
Elena J. Jakubiak, Ronald N. Perry, Sarah F. Frisken
}

TR2006-119 July 2006

\begin{abstract}
Because a typical Asian typeface can consist of more than 12,000 glyphs, traditional scalable outline-based fonts require 5-10 MBs of memory. This requirement is particularly problematic in mobile devices (e.g. cell phones and PDAs) and embedded systems (e.g. car navigation systems)where memory is at a premium. Existing commercial solutions (e.g. by Bitstream and Monotype Imaging) represent glyphs using simplified uniform-width strokes. However, these light-weigh ( $250 \mathrm{KBs}$ ) stroke-based fonts lack the detail, expressiveness, and variety needed for optimal legibility and true cultural acceptance (Figure 1). Although METAFONT [Knuth 1986] is stroke-based and provides sufficient detail and expressiveness, it requires the type designer to be proficient in mathematics, rasterization and programming.
\end{abstract}

ACM SIGGRAPH

This work may not be copied or reproduced in whole or in part for any commercial purpose. Permission to copy in whole or in part without payment of fee is granted for nonprofit educational and research purposes provided that all such whole or partial copies include the following: a notice that such copying is by permission of Mitsubishi Electric Research Laboratories, Inc.; an acknowledgment of the authors and individual contributions to the work; and all applicable portions of the copyright notice. Copying, reproduction, or republishing for any other purpose shall require a license with payment of fee to Mitsubishi Electric Research Laboratories, Inc. All rights reserved.

Copyright (C) Mitsubishi Electric Research Laboratories, Inc., 2006

201 Broadway, Cambridge, Massachusetts 02139 



\title{
An Improved Representation for Stroke-based Fonts
}

\author{
Elena J. Jakubiak* \\ Ronald N. Perry $\dagger$ \\ Sarah F. Frisken* \\ Tufts University \\ MERL \\ Tufts University
}

\section{Overview}

Because a typical Asian typeface can consist of more than 12,000 glyphs, traditional scalable outline-based fonts require $\sim 5-10 \mathrm{MBs}$ of memory. This requirement is particularly problematic in mobile devices (e.g., cell phones and PDAs) and embedded systems (e.g., car navigation systems) where memory is at a premium. Existing commercial solutions (e.g., by Bitstream and Monotype Imaging) represent glyphs using simplified uniform-width strokes. However, these light-weight $(\sim 250 \mathrm{KBs})$ stroke-based fonts lack the detail, expressiveness, and variety needed for optimal legibility and true cultural acceptance (Figure 1). Although METAFONT [Knuth 1986] is stroke-based and provides sufficient detail and expressiveness, it requires the type designer to be proficient in mathematics, rasterization, and programming.

In this presentation we describe an improved representation for stroke-based fonts, entitled Stylized Stroke Fonts (SSFs). SSFs overcome the shortcomings of existing scalable font representations by providing the detail and expressiveness of outline-based fonts with a memory footprint comparable to current stroke-based fonts. Each glyph of an SSF consists of three main elements: a stroke path which is composed of a set of line segments and Bezier curves and runs approximately along the centerline of the glyph; a set of stroke profiles which define the shape of the stroke by specifying the perpendicular distance from the stroke path to the edge of the glyph where the distances are interpolated along the length of the stroke path; and a set of stroke ends which determine the shape of the start and end of each stroke in the glyph (Figure 2). Stroke profiles and stroke ends are repeated both within individual glyphs and across multiple glyphs in a typeface. Stroke paths are repeated across typefaces. The modular SSF construction exploits this repetition and stores only one instance of each profile set, stroke end, and stroke path, thereby achieving the expressiveness of outline-based fonts with memory requirements that are only $33 \%$ larger than current stroke-based fonts (Figure 3).

SSFs leverage recent work in font representation and rendering using Adaptively Sampled Distance Fields [Frisken et al. 2000; Frisken and Perry 2006; Perry and Frisken 2005]. Adaptively Sampled Distance Fields provide very high quality anti-aliasing, a means for aligning glyphs to the pixel grid to improve legibility, simple and efficient operators for composing glyphs from multiple overlapping strokes, and direct rendering of SSF glyphs without having to first determine explicit outlines.
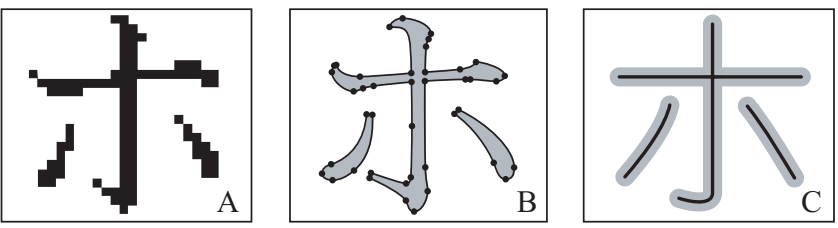

Figure 1. Common computer representations of glyphs include A) bitmaps, B) outlines, and C) strokes. Bitmaps give the best rendering at specific point sizes but are not scalable thus requiring many versions if multiple fonts and/or sizes are needed. Outlines are most expressive but can require $10 \mathrm{MBs}$ for Asian fonts. Stroke-based glyphs are scalable and much smaller ( $250 \mathrm{KBs}$ for Asian fonts) but are neither visually appealing nor expressive.

\footnotetext{
*e-mail: \{jakubiak, frisken\}@cs.tufts.edu

†e-mail: perry@merl.com
}

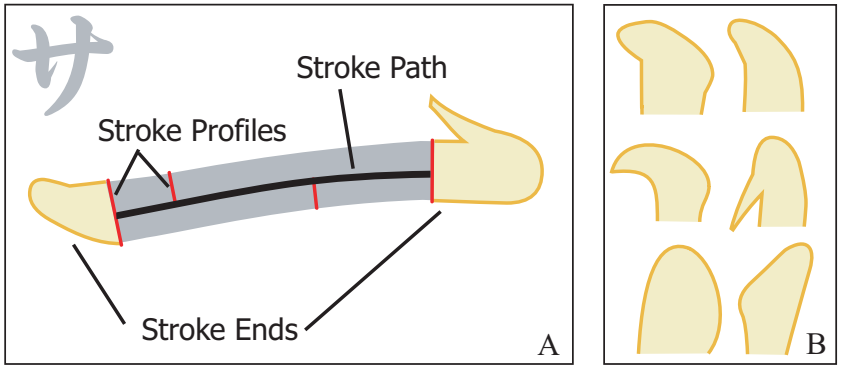

Figure 2 A) Glyphs in Stylized Stroke Fonts (SSFs) consist of stroke paths which run approximately along the centerline of the glyph, stroke profiles which specify the perpendicular distance from the stroke path to the edge of the glyph where the distances are interpolated along the stroke path, and interchangeable stroke ends which specify the shape of the ends of each stroke path. B) Various stroke ends.
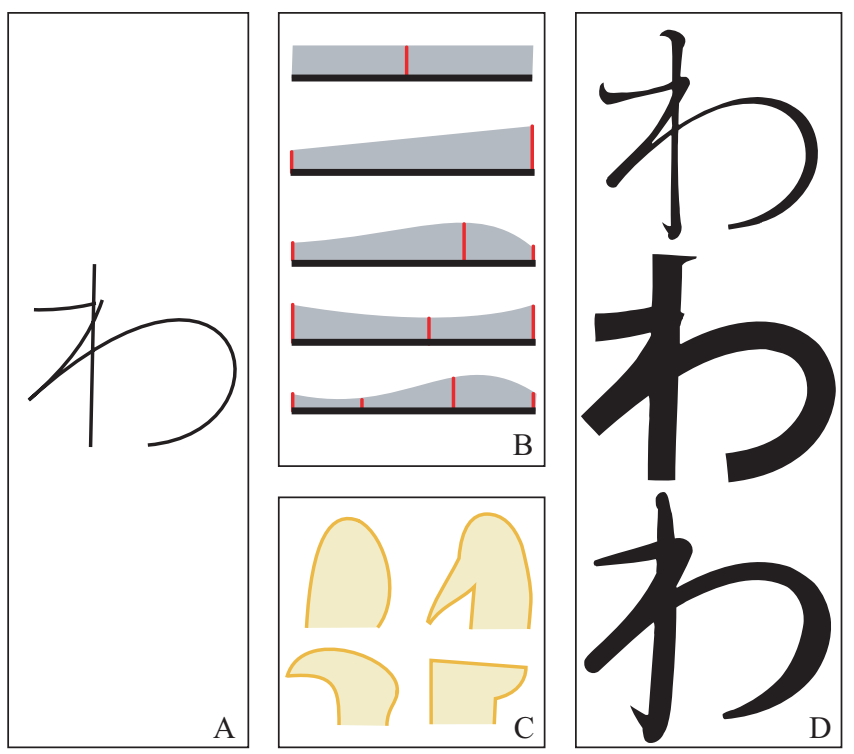

Figure 3. By combining A) a single stroke path with B) various stroke profiles, and C) various stroke ends, a rich variety of typefaces D) can be represented using very little memory. Because stroke profiles and stroke ends are repeated among glyphs both within and across typefaces, enhancing stroke-based fonts to represent multiple, expressive typefaces only requires an additional $80 \mathrm{KBs}$ over the $250 \mathrm{KBs}$ required for stroke-based Asian fonts.

\section{References}

Frisken, S., Perry, R., Rockwood, A., and Jones, T., 2000. Adaptively Sampled Distance Fields: A General Representation of Shape for Computer Graphics. Proc. ACM SIGGRAPH 2000, pp. 249-254.

Frisken, S. And Perry, R., 2006. U.S. Patent 7,002,598 “Generating a Composite Glyph and Rendering a Region of the Composite Glyph in Object-Order".

Knuth, D., 1986. The METAFONTbook. Addison-Wesley, USA. Perry, R. and Frisken, S., 2005. U.S. Patent 6,917,369 "Rendering Cell-based Distance Fields Using Texture Mapping”. 